\title{
Borradura de la identidad, heroísmo y suplantación en La carroza de Bolívar de Evelio Rosero y dos representaciones cinematográficas del héroe histórico ${ }^{1}$ latinoamericano
}

\author{
Ricardo Andrés Manrique Granados/Investigador independiente
}

\section{Resumen}

La recreaciones ficcionales recientes de la vida de Simón Bolívar han sido abundantes tanto en Colombia como en Venezuela, en donde han servido de estandartes políticos. ¿Qué procedimientos han usado para superar la brecha de lo histórico y ocupar lugares en los imaginarios sociales de ambos países? El presente artículo busca dirigirse hacia esa indagación a partir del estudio de La carroza de Bolívar (2012) de Evelio Rosero y de las películas de dos directores venezolanos sobre Bolívar. El marco crítico y teórico de Quentin Skinner permitirá una aproximación a las obras que otorgue un valor esencial a esta indagación organizada en torno al lugar de la ficción ante la historia y la cultura.

Palabras claves: Evelio Rosero, Simón Bolívar, identidad, historia, cine y literatura

\begin{abstract}
The recent fictional recreations of the life of Simón Bolívar have been abundant in Colombia and in Venezuela, where they have served as political banners. What procedures have used such productions to overcome the historical gap and occupy places in the social imaginaries of both countries? This article seeks to address this inquiry based on the study of La carroza de Bolivar (2012), by Evelio Rosero, and the films of two Venezuelan directors about Bolívar. The critical and theoretical framework of Quentin Skinner will allow an approach to these works that will provide an essential value to this inquiry organized around the place of fiction before history and culture.
\end{abstract}

Keywords: Evelio Rosero, Simón Bolívar, identity, history, cinema and literature
Múltiples han sido las aproximaciones que la producción cultural colombiana ha tenido en torno a la figura de Simón Bolívar. La novela de Gabriel García Márquez, El general en su laberinto (1989), y la película Bolívar soy yo! (2002), dirigida por Jorge Alí Triana, son solo dos ejemplos notables de las tentativas de representación del prócer latinoamericano que se han hecho en el país. Al igual que otras obras precedentes y posteriores, en sus propios lenguajes ambas se apropian de la imagen de Bolívar y conforman narrativas que, si bien corresponden por una parte a determinados aspectos utópicos del prócer y, por otra, a diversos rasgos históricos, evidencian matices con los que la figura de Bolívar ha sido representada, configurando perspectivas lúcidas ante problemáticas tales como los lugares de las artes en una sociedad convulsa y de la ficción ante la historia.

En tanto, múltiples han sido las apropiaciones de la figura de Bolívar que han sucedido en diversos contextos culturales. Como lo recuenta Nayibe Bermúdez Barrios en su estudio de 2008, la Base de Datos de Películas de Internet (Internet Movie Database) había registrado hasta el 2007 trece producciones cinematográficas de diversas naturalezas que habían tenido a Bolívar como protagonista, y además:

According to many academics, the cultural and historic significance of Bolívar has been tied up with his prestige (Jorge Melo 90), his mysticism and vision (Slatta 240,2061), his charisma and engaging writing style (Bushnell 13-14), his ideas for a centralized state (Jaramillo, "Nación" 114), as well as his qualities as a modern hero in whom both writing and power coalesce (González Echevarría 194). These views contrast, however, with other poignant images in which the Liberator appears as allied with the military-cleric elites (Bushnell 16-17; Jaramillo, "Regiones" 205), as a dictator (Jaramillo, "Regiones"; 204; Murray 294), as a victim of the fatherland (Potrowski 39), and as a flawed humanized figure (Cowie 34; Mendez Ramírez 199). All of this without counting the radically conflicting views presented by personalities as close to him as generals Daniel Florencio O'Leary and H.L.V. Ducoudray Holstein in their respective memoir (Archer). (Bermúdez Barrios 2008, 46)

Así pues, en medio de los matices inherentes a los diversos entornos culturales en los que se origina, la imagen de Bolívar ha ido anclada habitualmente a polaridades que 
corresponden bien a la desconfianza o a la admiración; al desencantamiento receloso o a la cuasi icónica fascinación: fenómeno que se ve reflejado, usualmente en su cariz más halagüeño, en las numerosas producciones fílmicas que han recurrido a él como protagonista. En ese sentido, si bien el presente artículo no propone un estudio detallado de las cintas que se han hecho en torno a Bolívar, ni busca analizar la totalidad de producciones literarias, críticas e historiográficas que se han gestado en torno de la figura del prócer - pues tales tentativas requerirían el análisis exhaustivo de una suma considerable de títulos como los aludidos anteriormente-, se reconoce de entrada la necesidad de un estudio de esa naturaleza y se busca conectar las distintas categorías textuales que atañen. Si bien el presente escrito no se niega a la posibilidad de ser eslabón de tal estudio, se limitará a proponer una perspectiva comparada que contempla por igual lo fílmico y lo literario, así como la producción dada en Colombia y Venezuela. En ese sentido, se trabajará en organizar un análisis de La carroza de Bolívar (2012) de Evelio Rosero, novela merecedora del Premio Nacional de Novela, y en articular un diálogo transdisciplinario con dos trabajos cinematográficos que vieron la luz en el 2013, y que fueron dirigidos respectivamente por los venezolanos Alberto Arvelo y Luis Lamata: Libertador y Bolivar, el hombre de las dificultades. ${ }^{2}$ Se buscará determinar de qué modos la novela del escritor bogotano Evelio Rosero obra que plasma las acciones de Bolívar en Pasto, baluarte de la resistencia realista, y sus alrededores-, se contrapone a las posibilidades de creación ficcional cinematográfica desplegadas en las dos cintas aludidas, en particular en cuanto dichas producciones se acoplaron en su momento a circunstancias sociopolíticas decisivas y que marcaron una coyuntura significativa para Venezuela, Colombia y América Latina.

En suma, el objetivo del trabajo será establecer contrastes y comparaciones relevantes que ayuden a aclarar el panorama del diálogo que se entabla en torno de la figura de Bolívar como el héroe latinoamericano: diálogo que rompe la brecha de lo nacional. Así, en primer lugar se analizarán algunos rasgos fundamentales de las cintas ante algunos precedentes historiográficos. En segundo lugar, se hará un estudio de la novela de Rosero que tiene en cuenta, no solo el análisis previo de los filmes, sino la relevancia historiográfica de obras de ficción como la del bogotano, de acuerdo con algunos preceptos teóricos de Quentin Skinner. Por último, se propondrán conclusiones provechosas para el estudio de un panorama cultural trasnacional en el que las comprensiones de lo heroico juegan papeles fundamentales en cuanto compete a lo político y lo social.

\section{Dos visiones cinematográficas del héroe latinoamericano ante su realidad textual}

Las contingencias políticas de la última década estimularon la aparición de modelos novedosos de apropiación de la figura bolivariana. El año 2013 marcó un cambio crucial en Latinoamérica. La muerte de Hugo Chávez, hasta entonces presidente de la República Bolivariana de Venezuela, supuso un conjunto de rupturas y de reorganizaciones políticas, económicas y sociales en el continente. ${ }^{3}$ Este hecho también tuvo repercusiones drásticas en la producción cultural; en particular, en la audiovisual, en la que novedosas interpretaciones significaron reapropiaciones de una imagen del Libertador que era, de por sí, problemática en términos de su caracterización y afiliación ideológica. En ese sentido, el surgimiento de nuevas concepciones de Bolívar fue propiciado, por una parte, por una globalización cambiante en la que Latinoamérica ha tenido un rol tan convulso como esencial. ${ }^{4}$ En ese escenario, lecturas deliberadamente parciales de sucesos históricos en los que el Libertador fue protagonista se ajustaron, por otra parte, a contextos económicos y sociales locales que ameritaron nuevas interpretaciones de la figura del héroe latinoamericano.

El Instituto Cubano del Arte e Industrias Cinematográficos y la Fundación Villa del Cine, complejo cinematográfico patrocinado por el gobierno de Venezuela, impulsaron la producción de Bolivar, el hombre de las dificultades (2013). La cinta, que se centra en las peripecias del prócer en los años 1815 y 1816, fue dirigida por el venezolano Luis Alberto Lamata (Film Affinity 2013a). El mismo año se estrenó la superproducción Libertador, que se propuso reconstruir la vida de Bolívar desde su etapa formativa hasta su declive. El largometraje, que fue dirigido por el también venezolano Alberto Arvelo, fue una coproducción de empresas privadas de Venezuela, España, Alemania y los Estados Unidos, pero recibió también el apoyo del Gobierno de Venezuela (Film Affinity 2013b). La película de Arvelo recaudó una suma considerable para cualquier producción latinoamericana realizada en ese momento pero, como veremos, se destacó también por las licencias con las que sus creadores se alejaron de los marcos históricos en que se desenvolvió la aventura transatlántica del Bolívar histórico.

Lo representado por Bolívar, el hombre de las dificultades se sitúa en un momento radicalmente significativo desde la perspectiva historiográfica. En 1815 Bolívar debió exiliarse en Jamaica, luego de que los ejércitos realistas retomaran el control de la Nueva Granada. Desde allí, el Libertador escribiría a Henry Cullen sobre los argumentos que justificarían la rebelión y, más adelante, la revolución en contra del Gobierno español.

En ese sentido, la película plantea un panorama en el que no solo se realza la oposición al Régimen español, sino que además se reitera la falta de apoyo de parte del Gobierno británico a la causa independentista. En ese sentido, desde un punto de vista historiográfico vale la pena indagar, primero, sobre por qué habría Bolívar de esperar tal apoyo estando situado, precisamente, en una colonia inglesa, y en una situación crítica tanto en el sentido político como en el económico, e incluso teniendo una salud en declive. La pregunta, en 
ese sentido, no versa solo acerca de la evidencia historiográfica que apunta a que el Libertador habría podido esperar semejante tipo de ayuda, sino la forma como podría ser verosímil dar mayor importancia a semejante causa, y no a la Realista. Es así notable que, precisamente en ese escenario, Bolívar redactara un documento que, centrándose en la opresión española, reclama el apoyo de otro Régimen: el inglés. En suma, es indispensable reconocer en cuál escenario, si bien el Libertador no obtuvo apoyo de ese Gobierno, sí fue capaz de despertar el interés de algunos británicos que se unieron a su causa, además de contratar mercenarios que defendieron su vida - aspecto que se estudiará progresivamente en el apartado dedicado al trabajo de Rosero-.

De acuerdo con las anteriores premisas, vale la pena remitirse directamente a la Carta de Jamaica (1915), y poner en un primer plano las alusiones que Simón Bolívar hizo a los derechos que correspondían a los nacidos en América. A saber:

Yo considero el estado actual de la América como cuando desplomado el Ymperio romano, cada desmembración formó un sistema político, conforme á sus intereses y situación, ó siguiendo la ambición particular de algunos Gefes, familiares ó Corporaciones. Con esta notable diferencia, que aquellos miembros dispersos volvían á restablecer sus antiguas naciones con las alteraciones que exigían las cosas ó los sucesos. Mas nosotros, que apenas conservamos vestigios de lo que en otro tiempo fue, y que por otra parte no somos Yndios ni Europeos, sino una especie media entre los legítimos propietarios del país y los usurpadores Españoles; en suma, siendo nosotros americanos por nacimiento; y nuestros derechos los de Europa, tenemos que disputar éstos á los del país, y que mantenernos en él contra la opinión de los invasores; así nos hallamos en el caso más extraordinario y complicado. (Bolívar 1915, 17)5

Con su alusión al modelo del Imperio Romano en su ocaso, Bolívar anticipa brillantemente el surgimiento de la concepción de América Latina como se la concibió desde el siglo XX. Sin embargo, también formula una premisa problemática cuando presenta a quiénes parece considerar 'verdaderos americanos', en oposición a los impostores que han venido a usurpar el poder y, en el mismo grado, a los auténticos acreedores del continente, a quienes sitúa en un segundo plano. En otras palabras, Bolívar parece rechazar vehementemente la intervención del europeo invasor pero, sorprendentemente, margina también la figura del indígena, a la que a su juicio corresponde un peso histórico tan prevalente en términos simbólicos como irrelevante en términos prácticos para la constitución de las Naciones americanas. En ese sentido, la película de Lamata además aborda brillantemente la temática de la exclusión de las mujeres y de los negros en la construcción de dichos proyecto continental, lo cual se escenifica con el viaje de Bolívar a Haití (Lamata 2013).

En contraste, de acuerdo con el Bolívar textual, el desarrollo de la historia, al igual que sus beneficios materiales, le son reservados al 'auténtico americano': al criollo, quien deberá cumplir el imperativo de descender de europeos para encajar en el paradigma impuesto. Aquella postura complementa su visión del saber según aspectos como el espacio, la demografía y la ciencia aplicada al conocimiento del continente:

En mi opinión, es imposible responder á las preguntas // folio $2 / /$ con que usted me ha honrado. El mismo Barón de Humboldt, con su universalidad de conocimientos teóricos y prácticos, apenas lo haría con esactitud; por que, aunque una parte de la Estadística y revolucion de América es conocida, me atrevo á asegurar que la mayor está cubierta de tinieblas, y por consecuencia, solo se pueden ofrecer conjeturas mas o menos aproximadas, sobre todo, en lo relativo á la suerte futura y á los verdaderos proyectos de los Americanos; pues cuantas combinaciones suministra la Historia de las naciones, de otras tantas és susceptible la nuestra, por sus posiciones físicas, por las vicisitudes de la guerra, y por los cálculos de la Política. (Bolívar 1915, 17)

De una manera tan sutil como ingeniosa, Bolívar otorga un lugar privilegiado al criollo; al americano, ilustrado y descendiente de europeos que cuenta con condiciones para apropiarse del territorio; al individuo cuyos privilegios le son otorgados por su formación, su saber, su origen, y por contar con la oportunidad de estar situado en el continente, es decir: por sus demás privilegios, que se condensan en un aspecto cognitivo que amerita la comparación con Humboldt. Mejor dicho, el fragmento hace notar que, ante las contingencias que envuelven a las gestas de independencia, el saber resulta esencial para la formulación discursiva del modelo de criollo que sustenta Bolívar, en la misma medida en que una cierta inconmensurabilidad del territorio impide el total conocimiento de América: excluye, así, incluso a europeos tan cultivados como Humboldt, e insinúa la puerta de entrada a una nueva identidad - la criolla - basada en el saber. En concordancia, Bolívar parece reiterar a lo largo de su escrito que únicamente los pertenecientes a cada una de las nuevas naciones americanas, quienes conocen el territorio y han luchado por él, podrán entablar acciones estratégicas para dominarlo (Bolívar 1915, 11-12). Así, el Bolívar histórico enaltece los esfuerzos de cada una de las regiones que resisten al dominio de los españoles mientras que otorga un lugar singular a los benefactores europeos:

La Europa haria un bien á la España en disuadirla de su obstinada temeridad, por que á lo menos le ahorraria los gastos que espende y la sangre que derrama; afin de que, fijando su atencion en sus 
propios recursos, fundase su prosperidad y poder sobre bases mas sólidas que de las de inciertas conquistas, un comercio precario, y esacciones violentas en pueblos remotos, enemigos y poderosos. La Europa misma por miras de sana politica, deberia haber preparado y ejecutado el proyecto de la Yndependencia Americana; no solo por que el equilibrio del mundo así lo exije, sino por que este és el medio lejitimo y seguro de adquirirse establesimientos ultramarinos de comercio. La Europa que no se halla agitada por las violentas pasiones de la venganza, ambicion y codicia, como la España, parese que estaba autorizada por todas las Leyes de la Equidad, á ilustrarla sobre sus bien entendidos intereses. (Bolívar 1915, 14)

El rol de la Europa benefactora apela al imperativo del libre intercambio para la gestación de un ordenamiento benéfico a ambos extremos del Atlántico, en cuyo caso se sugiere que la intervención del Viejo Continente habría sido oportuna en otro momento. Sin embargo, el grueso de la argumentación se dirige hacia la fiereza con que las poderosas naciones americanas han sido capaces de oponerse por sí mismas al régimen opresor. En ese sentido, el filme de Lamata se aleja notablemente de una realidad cuya disposición histórica y hermenéutica respalda tanto a intereses como a contingencias históricamente concentrados en las naciones americanas.

Distinto de lo que sugiere el filme de Lamata, el contacto de los criollos con las colonias inglesas - al igual que con las francesas - habría permitido un proceso de emancipación provechoso para las élites criollas, mas no para los indígenas ni para los esclavos afroamericanos. El acceso al poder habría quedado limitado desde sus raíces adscritas a esa parte de la población americana, y se habría vinculado con intereses privados, mas no con tentativas políticas institucionalizadas. En ese contexto, el fugaz pero intenso amorío que el Bolívar ficcional de Bolivar, el hombre de las dificultades comparte con Madamme Jeanne Bourvil, representaría esa tentativa incompleta de liberación auténtica, en la cual, además de los esclavos, eran incluidas las mujeres (Lamata 2013).

En contraste con la película de Lamata, Libertador se caracteriza por una heterogeneidad en su producción que distribuye mejor sus rasgos singulares y licencias creativas, al igual que sus desaciertos. Los desfases de la cinta de Arvelo se sitúan en el extremo opuesto de los de su coterránea, ya que enfatiza en la intervención de ideales e individuos extranjeros, mientras que pone en evidencia la volatilidad del protagonista mismo. De ese modo, las licencias del filme exponen la aparente crisis de personalidad de un Bolívar que desde el inicio del filme pone en evidencia una supuesta falta de independencia de su voluntad. Así, por una parte, el espectador habrá de ver a un Libertador que para desarrollar su empresa requiere del apoyo de Martin Torkington - personaje enteramente ficcional-: un supuesto inversionista inglés que habría alentado sus iniciativas. Por otra, la cinta enfatiza en la relevancia de un grupo de heroicos soldados provenientes de Gran Bretaña, entre quienes se encuentra el coronel irlandés James Rooke; mercenarios cuyo incondicional y casi suicida apoyo a la causa resulta inverosímil. En suma, la película de Arvelo se sitúa decididamente al otro extremo de la de Lamata: Libertador desdibuja al héroe que busca exaltar, pues precisamente toma mayores licencias ficcionales en intersticios históricos fundamentales; rasgos cuyas ausencias deforman el complejo heroísmo del Bolívar histórico - con todo y los cuestionamientos que pudieran surgir en torno a él- y, junto con él, los de los gestores del continente. Así, rebaja la importancia de la población americana, que supuestamente es el eje del discurso, y que efectivamente fue protagonista de la causa libertadora.

Por otra parte, el filme no solo se abstiene de mostrar las acciones arbitrarias de Bolívar contra aliados como José Prudencio Padilla o su poca gallardía en el campo de batalla - peculiaridad que Lamata tampoco reconoce-, sino que muestra en él una causa inverosímil. El Bolívar de Arvelo se entrega por un pueblo cuya importancia nunca es manifiesta, pero cuya imagen es reiterada constantemente, conformando con él un paisaje que ambienta unas intenciones épicas que nunca son completas en el filme: un aspecto en el que la interpretación de Lamata es mucho más afortunada, pues como lo evidencia el propio título, se centra los aspectos dificultosos y prácticamente inverosímiles de la causa libertadora. En ese sentido, la relación que el filme de Arvelo sugiere, existió entre Bolívar y Antonio José de Sucre, tampoco es verosímil, pues no trasciende los aspectos que suponen el origen privilegiado y las circunstancias históricas compartidos por ambos, de modo que los intereses políticos comunes quedan en un vago segundo plano; una carencia que parecería compensada por la materialidad americana que apenas insinúa una atribulada fotografía que, si bien muestra las dimensiones de la hazaña de la causa libertadora, rompe con toda verosimilitud. En consecuencia, el pesar del Bolívar de Arvelo por sus fracasos da en una lamentación por un ego herido; por una gran pérdida - la de la amada, que bien podría ser eco de María (1867) y así de un proyecto de nación inconcluso, un 'paisaje' (in)habitable y un letrado sabedor frustrado (ver Alzate 2011), que no se aprovechan sino que se agotan en grandilocuencia- y por una venganza fallida e imposible. En suma, aquella crisis de la personalidad da en una crisis de identidad, por la ausencia de rasgos que configuren un criollismo como el entrevisto previamente: un tópico que será abordado conjuntamente en el análisis de la obra de Rosero.

\section{La carroza de Bolívar: una respuesta escrita desde otra periferia}

Los ejércitos (2007), la novela por la que Evelio Rosero mereció el prestigioso premio Tusquets, comparte con $L a$ carroza de Bolivar (2012) un conjunto de rasgos formales y 
estructurales con los que vale la pena comenzar el abordaje de la obra del autor bogotano y sus consideraciones sobre el Libertador. Una referencia clásica, que alude al ingenio de Odiseo al enfrentar al cíclope Polifemo, da final a las desventuras de Ismael, protagonista de Los ejércitos que se ha sumido en los infiernos del trauma y la violencia en Colombia. Tras el delirio de la masacre, luego de haber conocido el horror absoluto por las circunstancias históricas de la agresión, la voz de Ismael profiere una frase desconcertante sobre lo que diría a quienes ve violar el cadáver de Geraldina, su hermosa vecina, que otrora admiró desde la distancia: "Les diré que me llamo Jesucristo, les diré que me llamo Simón Bolívar, les diré que me llamo Nadie" (Rosero 2007, 203).

En lugar de organizar en torno de sí una tabla de salvación, como en la Odisea, el gesto de Ismael de llamarse a sí mismo Nadie, derrumba simbólicamente todo sentido de humanidad que pueda quedar en él. Lo que en el canon de la literatura y del pensamiento occidentales fue ingenio o lucidez es, ahora, estupidez o locura; y lo que significó en la obra de Homero un escape ante la barbarie que representaba Polifemo, se transforma, en esta oportunidad, en un gesto de total rendición ante ella. ${ }^{6}$ Ahora bien, Rosero amplía los alcances de ese gesto al mencionar a Bolívar y a Jesucristo pues son dos figuras cuyos cánones de pensamiento - respectivamente al Catolicismo y la Patriaresultan obsoletos e incluso nocivos ante la agresión, pues la justifican. En suma, dicha alusión parece preludiar los gestos textuales que constituyen respectivamente Plegaria por un papa envenenado (2014) — novela que no será tratada en esta oportunidad, pues no corresponde con el eje de estudios, pero que cabe mencionar-, y La carroza de Bolivar, obra cuyo andamiaje ficcional encaja en las alusiones a los textos clásicos que se reconocen al final de Los ejércitos.

Desde su inicio, La carroza de Bolívar hace manifiesta su alusión a los textos clásicos. El uso de un recurso similar al que da cierre a Los ejércitos demarca la inmersión en la profunda temática de la ausencia y la crisis de la identidad, recurso que desentraña, no solo la complejidad historiográfica inherente a la temática de lo criollo y su régimen de saber y de exclusiones, sino la remisión a una ausencia de la posibilidad del conocimiento de ello y de lo propio: una pérdida del sentido del lenguaje, o de todo significado, en el marco de un fenómeno similar al que Derrida denomina una archi-escritura, y la aparición del significante del significante que suscita una borradura del nombre propio (Derrida 1981, 111). En ese sentido, al inicio de la novela de Rosero, una voz remite a la del rapsoda de los cánones clásicos; sin embargo, dicha voz narrativa parece cantar a su propia musa, y no a la de las obras clásicas griegas:

Ayúdame a desenterrar la sombra del doctor Justo Pastor Proceso López, a descubrir la memoria de sus hijas, desde el día que la menor cumplía siete años y la mayor era desflorada en el establo de la finca, hasta el día de la muerte del doctor, pateado por un asno en plena avenida, pero háblame también del extravío de su mujer, Primavera Pinzón, canta su amor insospechado, dame fuerzas para buscar el exacto día nefasto en que el doctor se disfrazó de simio, a manera de broma inaugural, resuelto a sorprender a su mujer con un primer susto de carnaval de Blancos y Negros, ¿qué día fue?, 28 de diciembre, día de Inocentes, día de bromas, día de agua y baño purificador, año de 1966. (Rosero 2012, 13)

A primera vista, la invocada parece ser Clio, musa de la historia y la epopeya; hija de Memoria encargada de las vidas de los héroes: un carácter coincidente con una noción de identidad, ahora ausente y difícil de recuperar, enmarcado ahora en la enunciación del tradicional carnaval de Blancos y Negros. Además, este llamado tiene un tono singular: un matiz que trasciende lo trágico y que se asocia con la comedia. El lector verá cómo en el desarrollo de la novela el fragmento citado opera como una expresión mínima que se amplía y magnifica fractalmente a lo largo del relato.

La historia de Justo Pastor es la narración del fracaso de una reescritura historiográfica que debería haber develado el semblante desconocido del héroe nacional: el rostro de un hombre no solo injusto, bajo y sanguinario, sino además cobarde y decepcionante como héroe. Así, la novela se organiza como la reconstrucción de un fracaso en la empresa de develar un fracaso. La derrota de Justo Pastor, quien es incapaz de mostrar la derrota de Bolívar, adquiere en ese sentido una importancia fundamental, pues organiza un tropo que, autocontenido, envuelve a la novela; y así permite la entrada del discurso literario en el historiográfico. En suma, como veremos, el trabajo de Rosero se inmiscuye, mediante el sentir cultural que demarca la tradición, en la historia, y además en la historia del presente.

En primera instancia, La carroza de Bolívar expone la cobardía de Bolívar en el campo de batalla; la crueldad con la que traicionó a aliados como José Prudencio Padilla, quien por demás fue discriminado por las élites criollas por motivo de su raza; y el ahínco con que promocionó su imagen como la del Libertador (Rosero 2012, 65-67), quizá, su único éxito según ese hilo discursivo. Sin embargo, la mayor virtud del relato se relaciona con circunstancias que, situándose en un contexto más local, hieren hondo en la historia del país. Así, Rosero evidencia el papel que Bolívar tuvo en la primera gran masacre de la Nueva Granada de entonces, hoy Colombia: masacre conocida como la Navidad Negra.

En aquel entonces, las huestes bolivarianas habían tenido que lidiar con la fiereza de la resistencia realista pastusa y con el líder indígena Agustín Agualongo. Sin embargo, para 1822, año de la masacre, el pueblo había desistido de las tentativas de rebelión. La masacre, narrada desde la perspectiva de Hilaria Ocampo, quien fuera víctima 
y eslabón reaccionario ante los excesos del Libertador, aporta una perspectiva lúcida de la confrontación:

- Cuando los hombres de Agualongo abandonaron la ciudad, ella creyó, como la mayoría, que si la resistencia había claudicado también la matanza se detendría, que Pasto habría sido tomada y de nuevo sobrevendría la paz, restringida, pero paz al fin [...] Lo que no sospechó fue la posterior acción de los asesinos del Rifles en la ciudad desprotegida: "La matanza de hombres, mujeres y niños, se hizo aunque se acogían a las iglesias, y las calles quedaron cubiertas de cadáveres; de modo que el tiempo de los Rifles es frase que ha quedado en Pasto para significar la cruenta catástrofe" nos dice Sañudo. (Rosero 2012, 216)

Para llegar a mostrar este suceso, en la novela se conectan varios ejes narrativos. Por una parte, Justo Pastor Proceso habla de su entrevista con Polina Agrado, heredera de los excesos de Bolívar. Y por otra, el relato da cuenta de la profunda investigación emprendida por el historiador nariñense José Rafael Sañudo. Con todo, lo verdaderamente novedoso del texto se sitúa en cómo ambos ejes convergen en las indagaciones del catedrático Arcaín Chivo: indagaciones que se basan, entre otros recursos, en las denuncias que Carlos Marx hace de la imagen del Libertador; acusaciones que llevan a formular profundos cuestionamientos de la imagen paradigmática de Bolívar como un 'líder generoso', valiente y desinteresado, y a afirmar en su lugar la figura del primer dictador latinoamericano (Rosero 2012, 216, 232). Sin embargo, como se explorará suficientemente más adelante, dichas perspectivas están provistas de sesgos que requieren ser analizados.

En tanto, cabe destacar que el texto organiza una nueva perspectiva ante hábitos culturales antes naturalizados. Los marcos no ficcionales y los ficticios convergen en la novela, de modo que realzan los sucesos históricos que dieron sustento al hábito cultural de la celebración del día de inocentes en Pasto - el carnaval-, desnaturalizando de paso la costumbre popular. En otras palabras, la novelación del suceso lleva a que el lector cuestione su comprensión de la feroz y cómica antesala del carnaval de Blancos y Negros. Se efectúa así una ruptura del hábito cultural y de la tradición por medio de la forma del relato. De ese modo, los marcos narrativos de la obra traen a un primer plano el profundo vacío histórico que acompaña tradiciones tan asentadas en nuestro país como la celebración de los carnavales de Pasto. Pero no es esta la única forma como la forma novelada de La carroza de Bolivar suscita profundas indagaciones históricas.

El diálogo que entabla Justo Pastor con el catedrático Arcaín Chivo, el alcalde Matías Serrano y el obispo monseñor Montúfar, origina un marco narrativo en torno al cual se reorganiza la percepción del lector del sujeto histórico. En ese sentido, es significativo que en la polifonía que permite el diálogo novelado de la obra converjan un representante de la academia, otro del clero y otro de la política gubernamental de la ciudad, y que a partir de la charla se desarrolle la desestabilización central de la figura de Bolívar. Lo mismo sucede con el hecho de que tal dinámica, que responde al gesto inicial de la invocación a las musas por una voz que muestre a profundidad las desgracias de Justo Pastor, desemboque en el fracaso total que evidencia el destino final de la carroza de Bolívar, que es destruida (Rosero 2012, 386). ¿Quizás ese gesto corresponda a una frustración histórica que atraviesa los orígenes del conflicto? A saber: ¿con la de la ausencia verdadera en los diálogos de los reales bandos beligerantes, y con cómo se vincula dicha ausencia con el desastre que da cierre a la obra? Tal noción corresponde, no solo al sentido de borradura y crisis de la identidad previamente anunciado, sino a un marco que dirige la atención hacia la historia del presente.

En consonancia, cobra relevancia el que, por una parte, la pandilla de estudiantes liderada por los hermanos Quiroz - el grupo que en primer lugar reprimió al catedrático Chivo - y, por otra, el general Lorenzo Aipe - amante de Primavera Pinzón, esposa de Justo Pastor-, quienes buscan desarticular la tentativa de reconstrucción histórica del doctor aglutinada en la carroza, estén siempre ausentes del diálogo sobre Bolívar, aunque sea a ellos a quienes más debería interesar el tema, pues son los agentes bélicos de la novela (Rosero 2012, 275-276). En ese mismo grado, es crucial que, a pesar de representar dos fuerzas aparentemente opuestas - a saber, la de un 'ejército' ultraconservador y la de una guerrilla mal fundamentada-, ambas organizaciones terminen compartiendo la causa de la defensa ciega del emblema bolivariano (Rosero 2012, 382). Dicha defensa se transforma, en consecuencia, en testimonio de una frustración histórica que trasciende al presente: representa el mismo sentido de impotencia de quienes continúan una guerra sin cuartel por causas difusas y, al estar al margen de las políticas gubernamentales, se organizan inefectivamente, caducan y fracasan. Pero, ¿cómo se contrastan estas perspectivas de la narración novelada escrita con ambas producciones fílmicas estudiadas, en especial según las peculiaridades de lenguajes y ritmos de difusión distintas, al igual que de lugares diferentes en los imaginarios colectivos?

Con el fin de abordar esta indagación, cabe remitirse a la alusión y el uso que Nayibe Bermúdez Barrios hace de los preceptos teóricos de Alison Landsberg, en el marco de su estudio de la película colombiana Bolivar soy yo. Como se observará, dichas nociones dirigen la atención hacia el lugar de la performatividad en la producción de Jorge Alí Triana: "By emphasizing the abilities of film to generate feelings such as empathy, Landsberg's model offers a way of linking emotions to values and prosthetic memory to political action. Landsberg's hypothesis seems productive for the study of Bolívar and the telenovela as ways of bridging gaps between individuals. In fact, Landsberg's study echoes 
Jesús Martín Barbero's and Sergio Cabrera's call for taking seriously the various technologies of memory, including film (Landsberg 158)" (Bermúdez Barrios 2008, 50). En su ensayo, Bermúdez Barrios se dedica al análisis de la película de Triana, en la que un actor de telenovela que personifica a Bolívar comienza a creer que es su personaje. Así, asocia la noción de la memoria prostética con las posibilidades que otorga lo cinematográfico, en tanto que la asignación colectiva de recuerdos o experiencias no vividos coincide con la imposición de un sentir común: una asociación que en el mejor de los casos se dirige, como se muestra con el foco de la telenovela en la producción de Triana, hacia la empatía y la indagación política (Bermúdez Barrios 2008, $60,62,63)$. Una práctica similar, pero trocada en su cariz más problemático, es la que se ha notado en el estudio de las producciones cinematográficas de Lamata y Arvelo: son reapropiaciones y reinterpretaciones de la historia que, a diferencia del desencantamiento político y bélico - con tintes barrocos - notado por Bermúdez Barrios en la película de Triana, dan en la exaltación de lo político y, consecuentemente, de lo bélico (ver Benjamin 2003, 96-98). ¿Pero no se extienden circunstancias paralelas igualmente inconvenientes a un contexto como el de la escritura novelada de Rosero?

En su ensayo, Bermúdez Barrios aborda también varias intervenciones y acciones del historiador colombiano Germán Arciniegas y Hugo Chávez en torno de la imagen de Bolívar; acciones que se vinculan con la noción de la performatividad (Bermúdez Barrios 2008, 46, 48, 49). "From this perspective, performance becomes one of the tools for the construction, transmission, and resignification of national identity" (Bermúdez Barrios 2008, 49). Dichas acciones políticas, con sus peligros e implicaciones, dirigen la atención hacia una instancia de performatividad individual; en tanto, la obra de Rosero aboca su bien planteada crítica de Bolívar en el contexto de un conjunto de tradiciones cuyo raigambre político no necesariamente corresponde con el de la obra, y en ello se encuentra su mayor desliz: en poner en contacto un sentido de identidad local y colectivo - en este caso, el de Nariño - como el carnaval, una práctica eminentemente performativa y colectiva, con una crítica histórica e historiográfica, notable por sus aspectos conflictivos, como la desarrollada en torno de Bolívar. En tanto, como era de esperarse por las diferencias de los lenguajes novelado y cinematográfico, el método en la narrativa del bogotano es diametralmente opuesto al de los directores venezolanos; pero además, mientras que ellos se concentran en un individuo cuyos rasgos humanos se acentúan e incluso se distorsionan, Rosero hace énfasis en sus actos, y en las consecuencias de estos en el imaginario de un país que solo ahora contempla algunas perspectivas que apuntan hacia el fin del Conflicto. Como se explorará, varias son las limitaciones que corresponden a dicha propuesta del lugar de la escritura literaria ante la historia y las prácticas culturales.

\section{A héroe muerto, héroe (im)puesto: una teoría de la novela histórica y la borradura de la identidad latinoamericana}

Como se ha expuesto con el análisis desarrollado, $L a$ carroza de Bolivar pone en evidencia en qué grado la ficción ocupa un nuevo lugar ante la historia. La novela ejecuta un acto de distanciamiento ante la concepción aristotélica según la cual la historia narra las cosas como fueron mientras que la poesía las muestra como pudieron haber sido (Aristóteles 2003, 1451b 73-74). La narrativa de Rosero, por el contrario, evidencia en qué medida la historia resulta ser a partir de un conjunto amplio de circunstancias y hechos: es, primero que todo, un relato múltiple, flexible y no lineal, para ser luego reinterpretado de distintas formas - en diversos momentos del decurso histórico - de acuerdo con la información disponible, y según las circunstancias políticas que la rodeen como narrativa. En consonancia, es notable cómo el historiador y teórico Quentin Skinner realza en sus textos la noción del contexto, dado que es esencial para el desarrollo de la historia de las ideas (Skinner 1969, 3).

Como Skinner advierte, el proceso de la narrativa y la configuración de las ideas relevantes para la historia se deriva de un conjunto de supuestos; de concepciones dadas por sentado, que no conforman - como lo concebiría una visión dialéctica de la historia - el campo adverso al del la movilización histórica, sino que son precisamente bases del cambio histórico. Dicho proceso se enmarca así en el campo discursivo que conforma la realidad y, generalmente, $h a$ sido la realidad tanto para la historia como para el historiador (Skinner 1969, 3-4).

Además, en "Interpretación, racionalidad y verdad", tercer capítulo de Visiones políticas (2002), ${ }^{7} \quad$ Skinner desarrolla su premisa central acerca del desarrollo de la historia de las ideas; le da fundamentos analíticos, y pone ese conjunto de concepciones en el plano de las creencias. Skinner evidencia que la falsabilidad de un conjunto de ideas no significa un abandono del sistema lógico que las rodea. A pesar de que dichas nociones puedan ser desmentidas en otro momento de la historia, según Skinner, deben seguir siendo vistas como fundamentales para comprender el decurso histórico, pues lo han moldeado en tanto que han sido supuestos vistos como verdad en distintos momentos de la historia (Skinner 2002, 30-31). En el contexto de Bolívar, tal concepción resulta iluminadora. Habrá que reconocer que, si bien es posible que Bolívar fuera esencialmente un cobarde o un traidor para cualquier individuo que no significara un avance en su plan dictatorial, también es fundamental reconocer primero que fue capaz de convencer a la mayoría de que no lo era. Lo mismo ocurre con el hecho de que a ojos de todos se hubiera transformado en el epítome del criollo ejemplar: una figura dispuesta a sacrificarse - como Cristo- por un conjunto de Naciones entonces - ficticias, - inexistentes. Así, aunque una evaluación cuidadosa de sus acciones llevara a considerarlo el primer 
gran dictador y no el primer gran héroe latinoamericano, tiene una gran relevancia el modo como (con)textualmente Bolívar forjó de sí y para sí una imagen que trascendió sus propias limitaciones (Rosero 2012, 203).

En este punto vale la pena citar directamente a Gabriel García Márquez o, mejor, hacer mención de la cita que Rosero hace de él. Esto nos permitirá contemplar su perspectiva y la del canon cultural en paralelo a la del autor bogotano, y conducirá a aportar algunas evidencias historiográficas que llevarán a contemplar y refutar en el mismo grado la visión del Nobel:

Así describieron Carlos Marx y José Rafael Sañudo, cada uno a su manera, al Libertador Simón Bolívar, basados en testimonios y documentos irrefutables. Lo que no impediría que muchos años después la pluma pluscuamperfecta del taumaturgo hechicero escribiera que el general Bolívar "siempre tuvo a la muerte como un riesgo profesional sin remedio. Había hecho todas sus guerras en la línea de peligro. Había hecho todas sus guerras en la línea de peligro, sin sufrir ni un rasguño, y se movía en medio de la línea de fuego contrario con una serenidad tan insensata que hasta sus oficiales se conformaban con la explicación fácil de que se creía invulnerable. Andaba sin escolta, y comía y bebía sin ningún cuidado de le que le ofrecían donde fuera [...] su desinterés no era inconsciencia ni fatalismo, sino la certidumbre melancólica de que había de morir en su cama, pobre y desnudo, y sin el consuelo de la gratitud pública”. (Rosero 2012, 166-167)

Antes de citar a García Márquez, y de nombrar las juiciosas y acertadas intervenciones de Sañudo y Marx, la novela de Rosero ha puesto en evidencia que Bolívar dimitió en 1830 tras temer una derrota - sin dar siquiera la batallaa manos del general Páez en Maracaibo (Rosero 2012, 165). Es esta una circunstancia bien conocida, incluso, por la historia oficial. Curiosamente, los sucesos que precedieron la deserción del Libertador fueron propiciadas por él mismo: fue Bolívar quien, además de ordenar la ejecución del almirante Padilla - quien por su condición racial no tenía el privilegio de un juicio-, conspiró en contra de Francisco de Paula Santander, quien sería su oponente en Venezuela (Rosero 2012, 164-165). Por poco se salvó el general Santander de correr la misma suerte que el valeroso almirante Padilla. Sin embargo, antes de renunciar al puesto que ya varios miembros del Congreso le habían instado a abandonar - pues su falta de lealtad a la causa, su ambición y su impredictibilidad eran bien conocidas-, Bolívar fue capaz de asegurarse de recibir una pensión anual que le permitiera marcharse al extranjero.

La actitud conspiradora de Bolívar, que lo aleja de la figura de gran estratega militar que la historia oficial ve en él, se nota en el modo como quiso engañar a la resistencia pastusa hablando de un supuesto "tratado entre Portugal, Francia e Inglaterra en que estas naciones se comprometen a una mediación armada entre la América y la España" (Rosero 2012, 175). Dicha tentativa solo tuvo como consecuencia que antiguos aliados como "José María Obando con otros cuatro oficiales se pasasen a los republicanos" (Rosero 2012, 179). En tanto, y a pesar de acciones evidentemente desacertadas para la causa independista, Bolívar fue capaz de hacer de sí mismo una figura tan respetada que obtuvo reconocimientos y homenajes que correspondían a otros miembros de la campaña, siendo ese el caso de la Batalla de Pichincha, cuyo verdadero acreedor fue "un joven y desconocido Antonio José de Sucre" (Rosero 2012, 200). Lo mismo sucedió con las Capitulaciones de Pasto, en cuyo caso dirigió una afanosa correspondencia a Santander "en que se advierte su ánimo de rebajar la victoria de Sucre" (Rosero 2012, 201). La carta es transcrita en la novela en su totalidad.

En este punto es relevante notar que las pocas aptitudes políticas y bélicas del Libertador tuvieron fuertes consecuencias en las campañas políticas y militares en las que intervino. Sin embargo, es igual de importante notar lo poco razonable que sería suponer que el autoproclamado Libertador y supuesto estratega militar no estableciera siquiera medidas de protección para sí mismo y que estuviera en la primera línea de batalla, como lo asegura García Márquez. En otras palabras, bien fuera Bolívar un proyecto de líder o de dictador, ¿desde qué ángulo sería aceptable tal comportamiento? En este punto vale la pena retornar al trabajo de Skinner.

El historiador y teórico inglés discute en varios de sus trabajos los grados de racionalidad con que se aceptan determinadas 'verdades históricas'. En ese contexto, juega un rol fundamental la noción de coherencia en torno a la que él trabaja, al igual que la concepción de racionalidad que estructura su pensamiento.

A rational agent will be someone whose beliefs are held in the light of a certain attitude towards the process of belief formation itself. This attitude must certainly include an interest in consistency. Rational agents want their reasons for holding their beliefs to bear upon their truth. But to espouse a given belief as well as its contradictory is to hold at least one belief that must be false. A rational agent will thus be concerned, at least in seriously troubling cases, to identify and eliminate any such obvious inconsistencies. Above all, rational agents must be interested in the justification of their beliefs. They must be concerned with the kind of coherence, and where appropriate the kind of evidence, that give them grounds for concluding that their affirmations of belief can be in fact justified. They will thus be concerned to view their own beliefs critically, to consider whether they really can be justified by considering the degree to which they may be said to 
fit with each other and with perceptual experience. (Skinner 2002, 32)

La crítica, de acuerdo con esta visión de la historia, existe; pero solo para considerar aquello que no es una creencia previa. En otras palabras: desde una perspectiva historiográfica, no se puede obturar un crítica real de la historia sino en ella, porque su criterio está limitado por determinadas creencias que anticipan la conclusión misma. Así, una visión como la de García Márquez corresponde con el aspecto de coherencia propuesto por Skinner. En ese contexto $-\mathrm{y}$ precisamente por ese contexto-, cualquier actuación, bien sea temeraria, cobarde o irracional, es tomada por valiente, ingeniosa o estratégica por lectores y escritores como García Márquez, solo porque quien la ha ejecutado, Bolívar - el héroe (d)escritor y (d)escrito-, ha creado condiciones textuales aptas para ser evaluado como el parangón de la virtud: de ahí el irónico y sucinto gesto textual que se observa en el texto de Rosero en Los ejércitos "Les diré que me llamo Jesucristo, les diré que me llamo Simón Bolívar, les diré que me llamo Nadie" (Rosero 2007, 203), que junta los arquetipos del héroe y el sacrificado. La imagen de aquel héroe abnegado ha sido delineada con tal cuidado que a primera vista se excluiría la posibilidad de que el medio en el que se enmarca sea verosímil; sin embargo, dado que este medio no incluirá sino que será el pueblo mismo - que habría de ser salvado por el caudillo y que se transformará en un aspecto instrumental de la construcción del tema épico-, el relato se hace historia. De ese modo, al igual que en las producciones de ambos directores venezolanos, en la novela de García Márquez el relato se hace fehaciente a costa de la verdad del héroe, que se desvanece como la del pueblo; pero son otros los procederes que imponen esta figura, y otras sus consecuencias. ¿Cómo abordar tales implicaciones?

\section{$* * *$}

Como se ha analizado de la mano de Quentin Skinner, la investigación histórica supone traer a colación consistentemente los supuestos que han alentado cualquier cambio histórico, para evaluar los papeles de cada individuo en el decurso de los hechos. Dichas nociones se atienen a un conjunto de fenómenos discursivos enmarcados en procesos contextuales de por sí significativos. Es posible afirmar que los rasgos historiográficos observados en las obras del Nobel y de ambos directores venezolanos, y sus contrastes, expuestos bajo el lente teórico propuesto con Skinner, son parciales en tanto que se invierte el orden historiográfico propuesto por el teórico inglés. De acuerdo con Skinner, los sujetos históricos deben ser analizados de tal modo que se estimen sus contextos y, de acuerdo con ellos, se determinen sus roles en la historia (Skinner 1969, 4-5). En contraste, aunque de maneras distintas, en la reconstrucción de García Márquez y en ambas obras cinematográficas analizadas la imagen de Bolívar y los rasgos de su supuesto heroísmo determinan la visión del contexto que rodea al personaje histórico. Sin embargo, la alternativa que se yergue en la obra de Rosero no está de exenta peculiaridades y deficiencias que deben ser contempladas.

Como se ha descrito previamente de la mano de Bermúdez Barrios y Landsberg, la ambientación del carnaval de Blancos y Negros y su asociación con la Navidad Negra en la novela da lugar a una cierta comprensión de la performatividad en la que se rompen los límites entre ficción y realidad, y se actualiza un sentir colectivo. Sin embargo, además se añade una carga narrativa en la que se borra al héroe que pudo haber sido Bolívar o su contendor, a costa de instaurar en su lugar al mártir, en Agualongo; o a la víctima, en Padilla; pero en cualquier caso no se formula una lectura histórica en la que verbigracia se expliquen las motivaciones de Agualongo para pertenecer a los Realistas, más allá de la oposición a Bolívar. Se cae de nuevo en el patrón de las polaridades y oposiciones que se enunció al inicio de este texto, y que es característico de las reconstrucciones del prócer (Bermúdez Barrios 2008, 46). Y en cualquier caso se formula una borradura de aspectos de una identidad como los formulados en torno al criollismo y sus vínculos con el saber: de nuevo, en la ficción como en la historia, la borradura es la identidad, mientras que la archiescritura permite la figuración de un tercer término o un significante del significante (Derrida 1981, 111). En suma, es esa suplantación de la historia el aspecto común de las narrativas estudiadas, tanto colombianas como venezolanas y tanto fílmicas como noveladas, aunque esos sean rasgos heredados de diversas narrativas y producciones que las preceden.

\section{Notas}

1. Un esbozo de la sección final del presente estudio, que se dedica a La carroza de Bolívar de Evelio Rosero, fue presentado en el XIX Congreso de la Asociación de Colombianistas. Colombia: Tradiciones y Rupturas.

2. Es importante anotar que Bolívar, el hombre de las dificultades fue también el nombre de una telenovela sobre el prócer dirigida por el colombiano Jorge Alí Triana, la cual fue transmitida en 1981 en Colombia y en 1983 en Venezuela. Triana se basó en la historia real de Pedro Montoya, el actor que protagonizó Bolivar soy yo! (Bermúdez Barrios 2008, 63).

3. Hugo Chávez fue presidente de Venezuela desde febrero de 1999 hasta marzo de 2013, año de su fallecimiento. Otra muestra de los contrastes y singularidades aludidos está en el texto de Blackmore (2017). 
4. Buena cuenta del problemático vínculo globalizado de los Estados Unidos con América Latina, y más específicamente, con Colombia, es la que se encuentra en los singulares pactos epistemológicos y materiales organizados en torno las aves según explica Quintero (2012): estudio que evidencia mediante el caso ornitológico que, aunque hay responsabilidad y ganancia de parte y parte en dichos intercambios, en ellos hay también profundos desbalances de poder.

5. En la transcripción de los fragmentos de la correspondencia de Bolívar se ha respetado, en todos los casos, la ortografía de los textos originales.

6. Se hace referencia al canto IX de la Odisea y al juego del lenguaje con el que Odiseo esquiva ingeniosamente a Polifemo al llamarse a sí mismo "Nadie".

7. Mis traducciones de ambos títulos.

\section{Obras citadas}

Alter Producciones Audiovisuales (Productores) \& Lamata Luis (Director). Bolívar, el hombre de las dificultades. 2013. Venezuela: Fundación Villa del Cine; Instituto Cubano del Arte e Industrias Cinematográficos (ICAIC); Wanda Films.

Alzate, Carolina. 2011. Otra amada y otro paisaje para nuestro Siglo XIX. Soledad Acosta de Samper y Eugenio Díaz Castro frente a María. Lingüistica y Literatura, No. 59: 117-135.

Aristóteles. 2003. Artes poéticas. Madrid: Visor.

Benjamin, Walter. 2003. La obra de arte en la época de su reproductibilidad técnica. Traducido por Andrés Weikert. México: Editorial Ítaca.

Bermúdez Barrios, Nayibe. 2008. Bolívar I am: Telenovela, Performance, and Colombian National Identity. Studies in Latin American Popular Culture, 27: 45-70

Blackmore, Lisa. 2017. Ruinas modernas y arte contemporáneo: el caso de El Helicode de la Roca Tarpeya. Cuadernos de Literatura, 21, No. 42: 255-277.

Bolívar, Simón. 1815 / 2015. Carta de Jamaica. Caracas: Comisión Presidencial para la Conmemoración del Bicentenario de la Carta de Jamaica.

Derrida, Jacques. 1981. Disseminations. Traducido por Barbara Johnson. Chicago: The University of Chicago Press.

Film Affinity. 2013a. Bolivar, el hombre de las dificultades. Recuperado de: http://www.filmaffinity.com/es/film339717.html

Film Affinity. 2013b. Libertador. Recuperado de: http://www.filmaffinity.com/es/film122108.html

Márquez, Gabriel García. 1989. El general en su laberinto. México: Oveja Negra.

Ochoa, Clara (Productora), \& Triana, Jorge Alí. (Director). 2002. Bolívar soy yo. Colombia: United Angels Productions.

Quintero, Camilo. 2012. Birds of Empire, Birds of Nation. Bogotá: Uniandes.

Rosero, Evelio. 2007. Los ejércitos. México: Tusquets.

Rosero, Evelio. 2012. La carroza de Bolívar. México: Tusquets.

San Mateo Films, WMG Film (Productores), \& Arvelo, Alberto (Director). 2013. Libertador. España \& Venezuela: Coproducción Venezuela-España; Producciones Insurgentes.

Skinner, Quentin. 1969. Meaning and Understanding in the History of Ideas. History and Theory, 8, No. 1: 3- 53.

Skinner, Quentin. 2002. Visions of politics. Cambridge: Cambridge University Press. 